PANCREATIC CANCER

\section{Fast or slow?}

Despite extensive research into pancreatic ductal adenocarcinoma (PDAC), the disease continues to have high mortality rates. The most widely accepted model of PDAC development is stepwise, involving sequential acquisition of independent mutations in several key oncogenes and tumour suppressors, leading to the development of aggressive disease from precursor lesions termed pancreatic intraepithelial neoplasia (PanIN). However, some evidence, such as the tendency for patients to present with metastatic disease and the high genomic instability of these tumours, suggest that progression might be more rapid than the accepted model implies.

Notta et al. analysed 107 PDAC whole genomes, first looking at causes of genomic instability. They developed an informatics tool to estimate ploidy and copy number, which has previously been difficult in PDAC owing to the high levels of fibrosis in these tumours. Approximately half of the tumours had evidence of polyploidization as well as high levels of copy number alterations (CNAs), indicating high genomic instability. Furthermore, mutational profiles suggested that most mutations occurred before polyploidization whereas most CNAs occurred after; CNAs were mostly or fully clonal within tumours, suggesting that they become dominant in the tumour and are likely

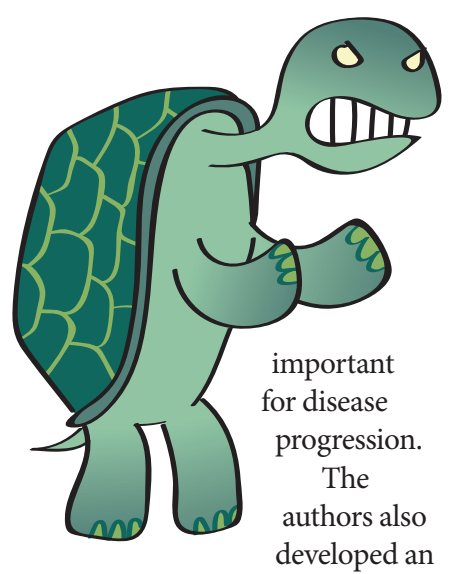

algorithm to differentiate chromothripsis from gradual genomic changes and found that at least one chromothripsis event happened in $\sim 65 \%$ of tumours. In addition, there was significantly more chromothripsis in polyploid tumours than in diploid tumours, and patients whose tumour had undergone chromothripsis had worse survival. A more detailed analysis of two individual tumours (one was polyploid and one diploid) indicated that more than half of CNAs in both cases could be attributed to chromothripsis, suggesting that catastrophic genetic events can have a substantial impact on PDAC genetics.

To understand the role of these events in disease progression, the authors then examined the genomes of metastatic lesions. In one case they sequenced eight distinct metastases from a patient. In this patient, chromothripsis preceded polyploidization; in all but one lesion (which underwent yet another chromothripsis event after metastasis), events characteristic of genomic instability occurred before metastasis. The dominant nature of this clone throughout nearly all metastatic lesions indicates that genomic instability tends to occur early and that the genetic changes acquired

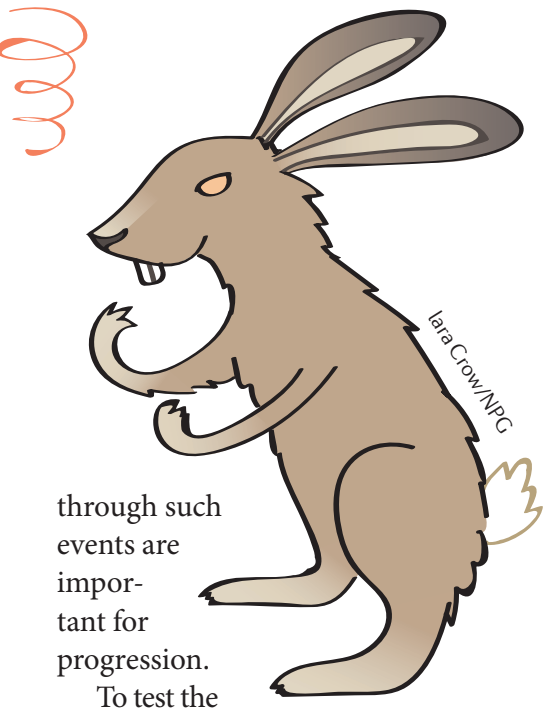

current gradual progression model using their data, the authors reconstructed the order of acquisition of mutations in PDAC driver genes (KRAS, cyclin-dependent kinase inhibitor 2A (CDKN2A), TP53 and SMAD4) in two tumours. In one, a single chromothripsis event combined with breakage-fusion-bridge cycles (another mechanism of genomic instability) was responsible for loss of one copy each of CDKN2A, TP53 and SMAD4; in the second, one chromothripsis event resulted in loss of CDKN2A and SMAD4. Overall, rearrangement patterns indicated that $16 \%$ of the 107 PDACs had combined allelic alterations in two or more of these genes. This suggests that at least some cases of PDAC do not follow the stepwise progression model.

These data challenge our view of PDAC progression and should be considered in the design of screening and therapeutic strategies for these tumours.

Sarah Seton-Rogers

ORIGINAL ARTICLE Notta, F. et al. A renewed model of pancreatic cancer evolution based on genomic rearrangement patterns. Nature $\mathbf{5 3 8}$, 378-382 (2016)

FURTHER READING Makohon-Moore, A. \& lacobuzio-Donahue, C. A. Pancreatic cancer biology and genetics from an evolutionary perspective. Nat. Rev. Cancer 16, 553-565 (2016) 